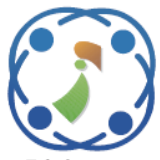

\title{
Defining Geometric Cross-Relevance Multivariate Trajectory Features for Transient Stability Analysis Based on Elastic Kernel Support Vector Machine
}

\author{
Seyed Alireza Bashiri Mosavi ${ }^{1 *}$ \\ Seyed Yashar Banihashem ${ }^{1}$ \\ ${ }^{1}$ Imam Khomeini International University \\ - Buin Zahra Higher Education Center of Engineering and Technology, Iran \\ * Corresponding author's Email: abashirimosavi@bzte.ac.ir
}

\begin{abstract}
Monitoring transient excursions of power system dynamic response is the most significant task for a reliable power supply. Hence, pursuing the best-laid features alongside a fit learning model for precise mining on the inherent characteristic of fast transient phenomena is considered a great concern on transient stability analysis (TSA). To this end, first, we discussed the root cause involved in selecting the cross-relevance (CR) features that influenced TSA. Furthermore, we regarded the geometric form of CR (GCR) features to measure the partial-whole manner of power system stability limits. Next, we triangulated Python technology, PSS/E, and MATLAB for generating the GCR multivariate trajectories. In the learning phase, to shroud the linear alignment weakness, the elastic kernel plugged into the support vector machine is considered for transient stability prediction (TSP). The results show an average accuracy near $97 \%$ with an acceptable average processing time of 691.7 milliseconds on test cases for TSP.
\end{abstract}

Keywords: Transient stability assessment, Multivariate trajectory features, Support vector machine.

\section{Introduction}

The power cascading events like generation and transmission grid failures challenge the secure power supply for all parties situated in the centralized or vertically infrastructure of the power system. This issue is fitted in the realm of power system stability problems. One of the main categories of power system stability studies is transient stability analysis (TSA) which focused on fast transient phenomena that negatively influenced the synchronous components of the power system. Transient stability refers to the ability of the power system to restore a stable state when subjected to severe-sudden disturbance [1]. Hence, precise monitoring of the transient swing curve can empower grid operators (GOs) for conducting accurate-timely control actions in severe transient space [2]. In this regard, transforming experiencedbased monitoring to smart-structured ones for raising the alertness of the GOs is the most significant issue for TSA. To this end, utilizing information technology (IT) capabilities in the service of electric power systems is followed as the strategic policy for an IT-driven power system. Developing the wide-area monitoring system (WAMS) based on phasor measurement units (PMUs) is a fundamental solution to achieving realtime TSA. However, data gathering via hardwarebased solutions cannot guarantee the optimum transient analysis. In fact, converting raw features (retrieved from PMUs) into the interrelatedness ones interlined with transient space should be regarded as a soft restructuring scheme. Furthermore, interlacing interdisciplinary fields including statistics, artificial intelligence, and machine learning as foundations of data mining (DM) technology to obtain the discriminative pattern for high-performance TSP is the next necessity in the soft restructuring scenario.

In recent years, the above-mentioned softoriented restructuring issues appeared on the agenda of scholars to solve the TSA concerns:

(a) Selecting features tightly related to the transient space is a preliminary concern to detect instability accurately. In fact, reasoned-based feature 
definition from power system component attributes has positively influenced the performance of TSA. Hence, several features have been considered by scholars in transient stability studies. In Reference [3], two types of features have been extracted for TSA by statistical properties of generator electromagnetic power and rotor angle trajectories. Reference [4] reports that post-fault observations on rotor angle and voltage magnitude caused the high prediction accuracy on transient analysis. The obtained results in $[5,6]$ show that relative angle measurement has a direct impact on TSP. Also, PMU-based monitoring focused on rotor angle excursions has been considered in Reference [7, 8]. In Reference $[9,10]$, the active power excursions are considered for TSA. Measuring voltage-frequency fluctuations against transient disturbance for TSA has been considered in Reference [11-13]. Also, reactive power variations in [14], and active power and voltage excursions in [15] are considered as selected features for TSA.

(b) The data mining techniques play a vital role in extracting discriminative patterns from inherent characteristics of transient data. The hyperplanebased algorithm mounted on the empirical risk minimization principle is the most widely used method in transient pattern recognition. In Reference [16], the support vector machine (SVM) has been considered as a predictor model in the presence of transient feature space including, voltages, frequencies, and rotor angles trajectories. Also, Reference [17] applied SVM for TSA in the 2684-bus Brazilian large-scale power system. Also, generator combinatorial trajectories entered to binary SVM as inputs for TSP in Reference [18]. The performance of soft margin SVMs for TSA depends on the RBF kernel functions on transient features such as kinetic energy of the generator, the average value of initial acceleration, and so on. Furthermore, the performance of soft margin SVMs for TSA based on the RBF kernel function applied on transient features such as kinetic energy of the generator, the average value of initial acceleration, and so on are considered in Reference [19]. Also, experimental comparison between kernels plugged into SVM versus other classification techniques has been considered for TSA in [20].

Regardless of proper solutions on dual aspects in previous researches (See Section 1; Paragraph (a) \& (b)), responding to unsolved challenges for more precise inference over the transient space has remained a major concern on TSP. These challenges include 1) In previous studies [3-13], transient analysis is based on the dynamic measurement of solid quantities of rotor angle, voltage, and so on. In fact, such an approach for feature definition cannot reflect the real condition of the power system. For example, transient stability status labeling via rotor angle excursion sometimes brings a different output in the real environment (we observed severe increases or decreasing the rotor angle, but the system is stable). Hence, we must focus on the root causes involved in feature definition that influenced the power network synchrony. Furthermore, partial measuring should be considered in features definition due to exposing part (versus the whole) of the power grid to transient instability conditions. In other words, the generalizability of defined features to measure the partial-whole manner of power system stability limits should be considered in transient analysis. In fact, defining cross-relevance features to record the effective excursions of transient sequences should be considered. This did not occur in previous research regarding (a) aspect. Hence, this issue motivated us to consider this challenge.

2) Focus on transient dataset characteristics in the formulations of the hyperplane-based predictive approach is a significant issue for high-performance TSP. In previous transient studies based on the SVM learning model [16-20] (See Section 1; Paragraph (b)), linear alignment has been considered by scholars as distance measuring between trajectorytype objects. In fact, applying kernel into SVM based on the point-to-point similarity index cannot compatible with the type of the transient feature space. Hence, using a proper mapping function that more compatible with multivariate trajectory feature space is the main factor transforming transient sequences to the separable decision surface.

According to raised proposed solutions to achieve optimum TSP, the definition of the geometric cross-relevance (GCR) multivariate trajectory features from the power system component attributes is considered as the primary contribution in this paper. Also, designing a reliable programming platform which is triangulated Python technology, SIEMENS power system simulator for engineering (PSS/E) application program interface (API), and matrix laboratory (MATLAB); we called PSPAM, to generate the GCR multivariate time series features through offline transient simulations which able to ensure compliance or mixing with the online application (online phasor measurement unit data) is an extreme necessity in data gathering phase. Finally, according to the proper performance of SVM classifier with point-to-point alignment on TSP in previous studies, applying SVM with nonlinear alignment on transient multivariate time series 
features is regarded as a great incentive to achieve high performance.

The paper is organized as follows: we elaborate on the definition of the GCR features for TSA in section 2. Next, a programming platform based on triangulated Python technology, SIEMENS PSS/E APIs, and MATLAB is offered for generating the GCR multivariate trajectory features. In the last part of this section, plugging elastic kernel into SVM classifier for optimal alignment among GCR trajectories is considered in training and testing procedures on TSP. Experimental design for evaluation of proposed framework based on cross validation-technique is presented in section 3 . Finally, section 4 depicts the conclusion of the paper.

\section{Methodology}

The proposed scheme for offline application as a parallel companion during the online procedure of real-time TSA is shown in Fig. 1. In this paper, three-step is considered as follows: 1) Discussion to define GCR features based on power system component attributes, 2) Transient dataset construction of GCR multivariate time series features based on proposed programming platform, namely PSPAM, and 3) plugging elastic kernel into SVM for optimal matching between transient sequences leading to high-performance TSP.

\subsection{Definition of GCR features for TSP}

Discussion on attributes of the power system components for mining behavior of transient data plays the driven-oriented role in TSA studies. Furthermore, reflecting on the cross-effect of the power system components attribute leads to better monitoring of power system transient behavior. In the following, we will describe this issue.

Loss of network synchrony begins with mechanical and electrical power imbalance of generators, variation in rotor angle based on imbalanced ratio, and mechanical characteristics of rotor related to the dynamic equation regulating its motion. In fact, the rotor angle instability derives from the rotor angle fluctuation (increasing or sometimes decreasing the rotor angle) in a way that is out of control. Such fluctuations are due to the imbalance between the input power and the output power of the generator. Now the question arises as to what proportion this imbalance increases or decreases the angle? The Inertia constant of a generator. The formulation of the torque of the generator $T$ in Eq. (1) is calculated by multiplying the $\delta_{\mathrm{a}}$ and $H$ where $H$ is the inertia constant of the generator, and $\delta_{\mathrm{a}}$ is the second derivative of the rotor angle (acceleration):

$$
T=\delta_{a} \times H
$$

On the other hand, the lack of an active power supply exacerbates the imbalance mentioned above. In a power grid, each bus voltage is a sinusoidal function according to (2):

$$
\vartheta(t)=V_{m} \cos \left(\omega t+\theta_{\vartheta}\right)
$$

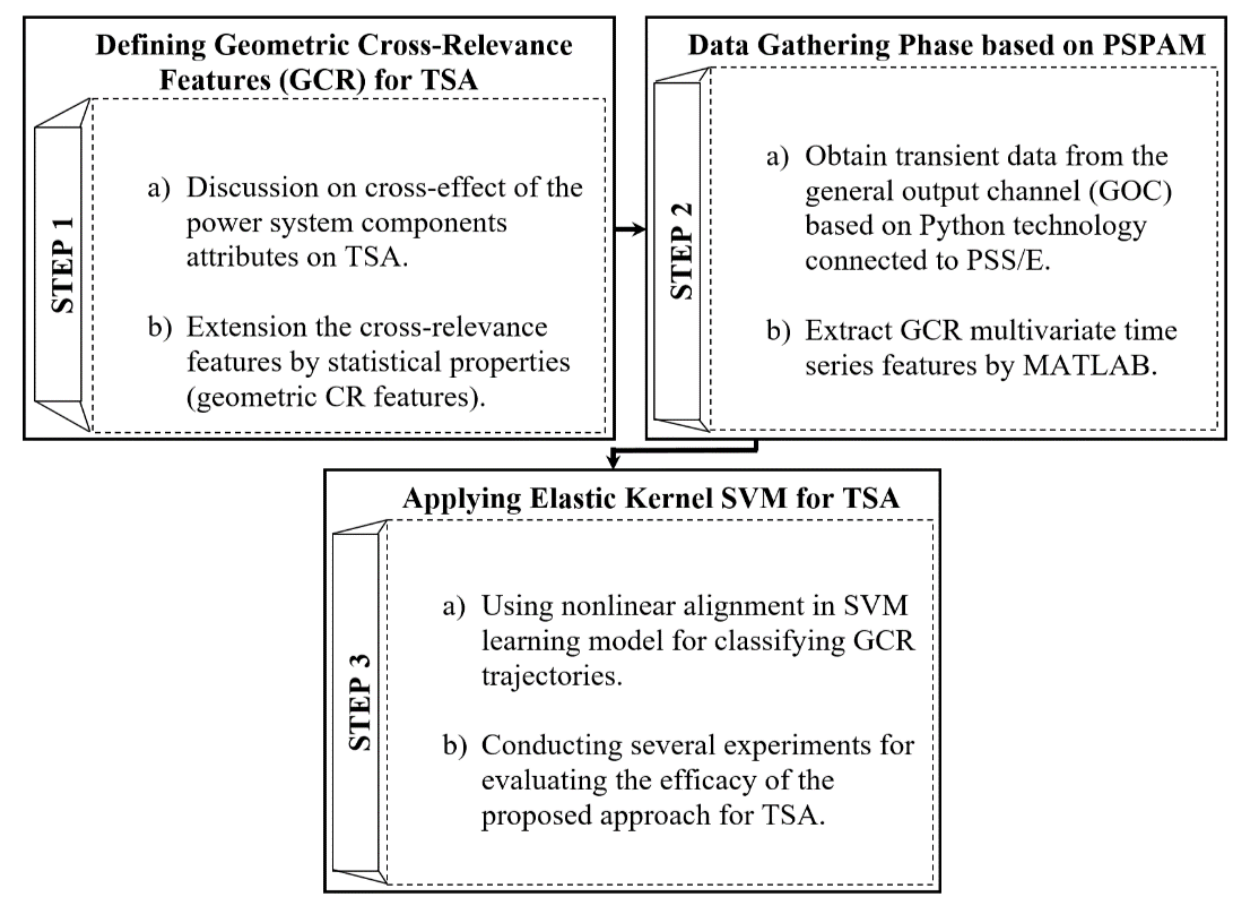

Figure. 1 Proposed framework for offline application on real-time TSA 
Where, the peak amplitude $V_{m}$, and the phase angle $\theta_{\vartheta}$ of the sinusoidal supply. Now we can represent such a voltage that changes sinusoidally on a complex plane with a vector. A vector whose length is $V_{m}$ and whose angle to the real axis is $\theta_{\vartheta}$, which we call voltage amplitude and voltage angle, respectively. If the mentioned vector starts to move on the complex plane and rotates at an angle of $\omega$, then its image will be $\vartheta(t)=V_{m} \cos \left(\omega t+\theta_{\vartheta}\right)$ on the real axis at all times.

Now consider the following scenario: suppose we have two busbars in direct current (DC) voltage. If the voltages of the two busbars are equal, no current will flow in the DC voltage. Because the subtraction value of the voltages is equal to zero. But in a power network based on alternating

Table 1. List of GCR 22-variate time series features

$$
\begin{aligned}
& F_{1}^{t_{m}}=\frac{\sum_{i=1}^{N_{\text {bus gen }}}{ }^{\text {PELEC }} C_{i}}{\sum_{i=1}^{N_{\text {bus gen }}} H_{i}} ; m=1.2 . . . l \\
& F_{2}^{t_{m}}=\operatorname{Max}\left(\left[V A N G L E_{i}\right]^{i=1: N_{b u s}}\right) ; m \\
& =1.2 \ldots . . . l ; \quad \text { slack bus }=0 \\
& \left.F_{3}^{t_{m}}=\operatorname{Min}\left([\text { VANGLE }]_{i}\right]^{i=1: N_{b u s}}\right) ; m \\
& =1.2 . \ldots l ; \quad \text { slack bus }=0 \\
& F_{4}^{t_{m}}=\operatorname{Var}\left(\left[V A N G L E_{i}\right]^{i=1: N_{\text {bus }}}\right) ; m \\
& =1.2 . . . . l ; \quad \text { slack bus }=0 \\
& F_{5}^{t_{m}}=\operatorname{Max}\left(a b s \left[V A N G L E_{i}\right.\right. \\
& \left.\left.-V A N G L E_{j}\right]^{i . j=1: N_{b u s}}\right) ; m \\
& =1.2 \ldots . l \\
& F_{6}^{t_{m}}=\operatorname{Mean}\left(a b s \left[V A N G L E_{i}\right.\right. \\
& \left.\left.-V A N G L E_{j}\right]^{i . j=1: N_{b u s}}\right) ; m \\
& =1.2 \ldots . . l \\
& F_{7}^{t_{m}}=\operatorname{Var}\left(a b s \left[\operatorname{VANGLE_{i}}\right.\right. \\
& \left.\left.-V A N G L E_{j}\right]^{i . j=1: N_{b u s}}\right) ; m \\
& =1.2 \ldots . l \\
& F_{8}^{t_{m}}=\frac{\sum_{i=1}^{N_{\text {bus gen }}} Q E L E C_{i}}{\sum_{i=1}^{N_{\text {bus gen }}} \text { Qmax }_{i}} ; m=1.2 . . . . l \\
& F_{9}^{t_{m}}=\operatorname{Max}\left(\left[\frac{Q E L E C_{i}}{Q \max _{i}}\right]^{\left.i=1: N_{\text {gen bus }}\right) ; m=1.2 . . . . l}\right. \\
& F_{10}^{t_{m}}=\operatorname{Min}\left(\left[\frac{Q E L E C_{i}}{\max _{i}}\right]^{\left.i=1: N_{\text {gen bus }}\right) ; m=1.2 . . . . l}\right. \\
& F_{11}^{t_{m}}=\operatorname{Var}\left(\left[\frac{Q E L E C_{i}}{Q \max _{i}}\right]^{i=1: N_{\text {gen bus }}}\right) ; m=1.2 . \ldots . l \\
& F_{12: 22}^{t_{m}}=\text { gradient of the } F_{1}: F_{11}
\end{aligned}
$$

Symbol: $N_{\text {bus gen }}=$ number of bus generator in test case, $Q E L E C=$ machine reactive power $(\mathrm{pu})$,

$Q \max =$ maximum amount of machine reactive power, $N_{\text {bus }}=$ number of buses, VANGLE= voltage phase angle, Var $=$ variance, Max $=$ maximum, Min $=$ minimum. current (AC), we can have two voltages with the same amplitude but with different angles, in which case the difference between these two voltages is not equal to zero, and the variable that causes power to flow in different lines is the difference in angle between voltages. In fact, it is the angle difference that controls the current active power in different parts of the power grid. As a result, active power is related to the voltage angle of the busbars, and also instability is related to the active power, so based on transitive closure, instability will be related to the voltage angle of the busbars.

As you know, not all elements in power systems are resistors. Resistors always consume power, which consumes real power called active power but capacitors and inductors do not consume power (average consumption). In an electrical periodic time, capacitors and inductors gain power twice and return the same power. It does not hold anything within itself to consume, nor does it produce anything. However, when we have capacitors and inductors in the network and apply voltage to it, at the moment it consumes power, we must give it power, and at the moment it produces, we must take power from it. Generally, these exchanges are redundant. Also, the amount of reactive power displacement in transmission lines affects the capacity of transmission lines. In addition to affecting transmission lines, it also causes losses in the power network.

An important point to note is that situating part of the power grid at stability limits (versus the whole) may cause transient instability. Hence, partial measuring in such conditions by regarding geometric functions like maximum, minimum, or variance values can provide proper statistics in the form of partial-whole manner power system monitoring. Hence, statistical descriptions of CR features called geometric CR (GCR) features were considered in feature expressions.

According to what was mentioned above, GCR 22-variate time series features are shown in Table 1.

\subsection{Transient dataset generation based on GCR features}

The proposed programming platform (PSPAM) is shown in Fig. 2. Generally, the PSPAM suite including two parts: a) Dataset construction module based on basic attributes (e.g., active power, voltage phase angle, and so on) and b) GCR 22-variate time series feature extraction module. In the first part of PSPAM architecture, we construct a dataset based on basic attributes by Python-based commands 
Table 2. List of PSS/E APIs-Python syntax based on 'psspy' module

\begin{tabular}{|c|c|c|}
\hline Disturbance & Description & Disturbance main arg. ${ }^{\text {a }}$ \\
\hline dist_bus_fault & $\begin{array}{l}\text { apply a fault at a bus during dynamic } \\
\qquad \operatorname{sim}^{\mathrm{b}}\end{array}$ & $\begin{array}{l}\text { ibus: \# bus which the fault is to be placed; } \\
\text { basekv: base voltage in } \mathrm{kV} \text { used to calculate } \\
\text { the per unit fault admittance. }\end{array}$ \\
\hline dist_branch_fault & $\begin{array}{c}\text { apply a fault at the IBUS end of a } \\
\text { non-transformer branch or a two- } \\
\text { winding transformer during dynamic } \\
\text { sim. }\end{array}$ & $\begin{array}{l}\text { ibus: \# bus of the bus at which the fault is to } \\
\text { be placed; jbus: \# bus of the other bus; } \\
\text { basekv: base voltage in } \mathrm{kV} \text { used to calculate } \\
\text { the per unit fault admittance. }\end{array}$ \\
\hline dist_bus_trip & $\begin{array}{l}\text { disconnect a bus during dynamic sim. } \\
\text { set a non-transformer branch or a }\end{array}$ & ibus: \# bus to be disconnected. \\
\hline dist_branch_trip & $\begin{array}{l}\text { two-winding transformer to out-of- } \\
\text { service during dynamic sim. } \\
\text { clear a fault during dynamic sim. }\end{array}$ & $\begin{array}{l}\text { ibus: \# bus of one of the buses; jbus: \# bus of } \\
\text { the other bus. }\end{array}$ \\
\hline \multicolumn{3}{|l|}{ dist_clear_fault } \\
\hline Output channel & Description & Output channel main arg. \\
\hline chsb & $\begin{array}{l}\text { specify the simulation variables to } \\
\text { monitor during dynamic sim. run } \\
\text { (activity chsb) }\end{array}$ & $\begin{array}{l}\text { status: an array of six elements (main index } \\
\text { of status array; } \\
\text { e.g. status }[5]=2 \text { for PELEC machine. }\end{array}$ \\
\hline Dynamics simulation & Description & Dynamics simulation function main arg. \\
\hline dynamic_solution_param_2 & $\begin{array}{c}\text { modify the dynamic sim. solution } \\
\text { parameters in dynamics working } \\
\text { memory. }\end{array}$ & $\begin{array}{l}\text { intgar: array of eight elements (main index of } \\
\text { intgar array; e.g.; intgar [1] for network } \\
\text { solution maximum number of iterations and } \\
\text { intgar [2] for number of output channels } \\
\text { being monitored). realer: array of eight } \\
\text { elements (main index of realer array; e.g.; } \\
\text { realer [2] for convergence tolerance used in } \\
\text { the network and realer [3] for simulation time } \\
\text { step). }\end{array}$ \\
\hline
\end{tabular}

dyre_new read a dynamics data file, and place the model references specified on its data records into dynamics working memory (activity DYRE).

\begin{tabular}{|c|c|c|}
\hline Power flow operation & Description & Power flow operation main arg. \\
\hline conl & $\begin{array}{l}\text { convert the constant MVA load for a } \\
\text { specified grouping of network loads } \\
\text { to a specified mixture of the constant } \\
\text { MVA, constant current, and constant } \\
\text { admittance load characteristics }\end{array}$ & $\begin{array}{l}\text { an array of four elements. the value of each } \\
\text { element is percent of the load being } \\
\text { converted (loadin [1] is the percent of active } \\
\text { power load to be converted to the constant } \\
\text { current characteristic; loadin [2] is the } \\
\text { percent of active power load to be converted } \\
\text { to the constant admittance characteristic.; } \\
\text { loadin [3] is the percent of reactive power } \\
\text { load to be converted to the constant current } \\
\text { characteristic; loadin [4] is the percent of } \\
\text { reactive power load to be converted to the } \\
\text { constant admittance characteristic. }\end{array}$ \\
\hline tysl & $\begin{array}{l}\text { convert generators from their power } \\
\text { flow representation in preparation for } \\
\text { switching studies and dynamic } \\
\text { simulations. } \\
\text { calculate a sparsity preserving } \\
\text { ordering of buses in preparation for } \\
\text { the processing of network matrices. } \\
\text { run switching study network } \\
\text { solutions. }\end{array}$ & \\
\hline
\end{tabular}

Symbols: [a: arguments; b: simulation] 
which support the various function of the SIEMENS PSS/E. As can be seen in Fig. 2, the first part including four steps which include characterizing sample operating conditions, run load flow, characterizing sample transient disturbance, and save the general output channel (GOC). Next, the PSSPLOT extension module as a link between these two steps restructuring the GOC in column-face format (cGOC). In the second step, the cGOC is used to extract the multivariate features by matrix laboratory (MATLAB). For more information on the proposed suite, refer to the following sections (Section 2.2.1- Section 2.2.3).

\subsubsection{Interaction python with $\mathrm{PSS} / \mathrm{E}$}

In this paper, the SIEMENS PSS/E software is used for transient simulation. The capabilities of $\mathrm{PSS} / \mathrm{E}$ show that the PSS/E is ideally suited for solving challenges of power system analysis (PSA). Also, PSS/E acts as a code-oriented model based on its APIs on Python technologies for PSA. In this section, a brief explanation of SIEMENS PSS/E API routines, their functions, and some of their important arguments by open Python API calls are followed. The execution of the transient simulation in PSS/E is formed through the 1) Power flow operation, 2) GOC, 3) Dynamic simulation, and 4) Disturbance APIs. As a concise guide of the PSS/E 33.4 API manual [21], Table 2 contained these functions to run simulations in PSS/E using the Python programming language.

\subsubsection{PSSPLOT extension module}

In this paper, we used a plotting program based on the Python extension module called PSSPLOT (or PSSPLT) to report the GOC file in a structured manner. Using PSSPLT functional program modules is helpful to perform data manipulation in the machine learning process. The PSSPLT procedure is handled in two ways: 1) Graphical user interface (GUI) operation, and 2) Batch operation. We selected the Batch operation for restructuring the GOC file (cGOC). According to the PSSPLT program manual [22], the activities of PSSPLT descriptions are shown in Table 3.

\subsubsection{GCR 22-variate time series feature extraction module}

First, After the data-gathering phase based on transient simulation mounted on the PSS/E API routines and columned arrangement of GOC file through the PSSPLT activities, the obtained transient dataset becomes GCR multivariate time
Table 3. PSS/E 33.4 PSSPLT activity descriptions

\begin{tabular}{ll}
\hline Batch operation of PSSPLT activities \\
\hline Activity & \multicolumn{1}{c}{ Description } \\
\hline CHNF & $\begin{array}{l}\text { Select binary response file activity }\left(^{*} \text {.out }\right. \\
\text { file: dynamic behaviour Activity obtained } \\
\text { from CHSB activity)). }\end{array}$ \\
POPT & $\begin{array}{l}\text { Plotting option setting activity. } \\
\text { PRNT }\end{array}$ \\
& $\begin{array}{l}\text { The channel tabulation activity to construct } \\
\text { a tabular listing of the values retrieved } \\
\text { from channels at each time step. }\end{array}$ \\
STOP & $\begin{array}{l}\text { Termination activity is used for normal } \\
\text { exit from PSSPLT. }\end{array}$ \\
\hline
\end{tabular}

series features (See Table 1) by MATLAB. In this step, we combine basic attributes along with their statistical descriptions (discussed in Section 2.1) to extract GCR trajectories. In fact, the task of this module is to construct the 22-variate time-series feature for feeding the machine learning procedure for transient stability status prediction. For example, the machine parameters were considered for finding the Inertia constant of a generator $H$ as the denominator in the case of the first univariate feature (See Table 1). To this end, the dynamic data file 'dyr' of the test system was addressed based on machine models such as the round rotor generator model (GENROU) [23]. For the rest of the univariate features depending on required parameters, such an approach is conceivable. Finally, the generated transient database is depicted in the form of Fig. 3.

\subsection{Applying learning model on transient dataset}

In this paper, we used the support vector machine (SVM) classifier as a learning model in training and testing procedures for TSP. Also, regarding the gathered contingency samples in trajectory-face form, plugging nonlinear alignment into the SVM

classifier formulation is considered in this section. After stating the principle of the support vector machine, the necessity of employing trajectory-specific kernel in SVM was considered in this section. More details on the learning model are described in Section 2.3.1 and Section 2.3.2.

\subsubsection{SVM classifier principle}

Support vector machine (SVM) was originally designed to be a binary classification, and it follows the principle of structural risk minimization without overfitting training data $[24,25]$. Because of this policy, the application of SVM in real-time TSA has been considered by scholars which is acceptable performance on transient stability status prediction. 


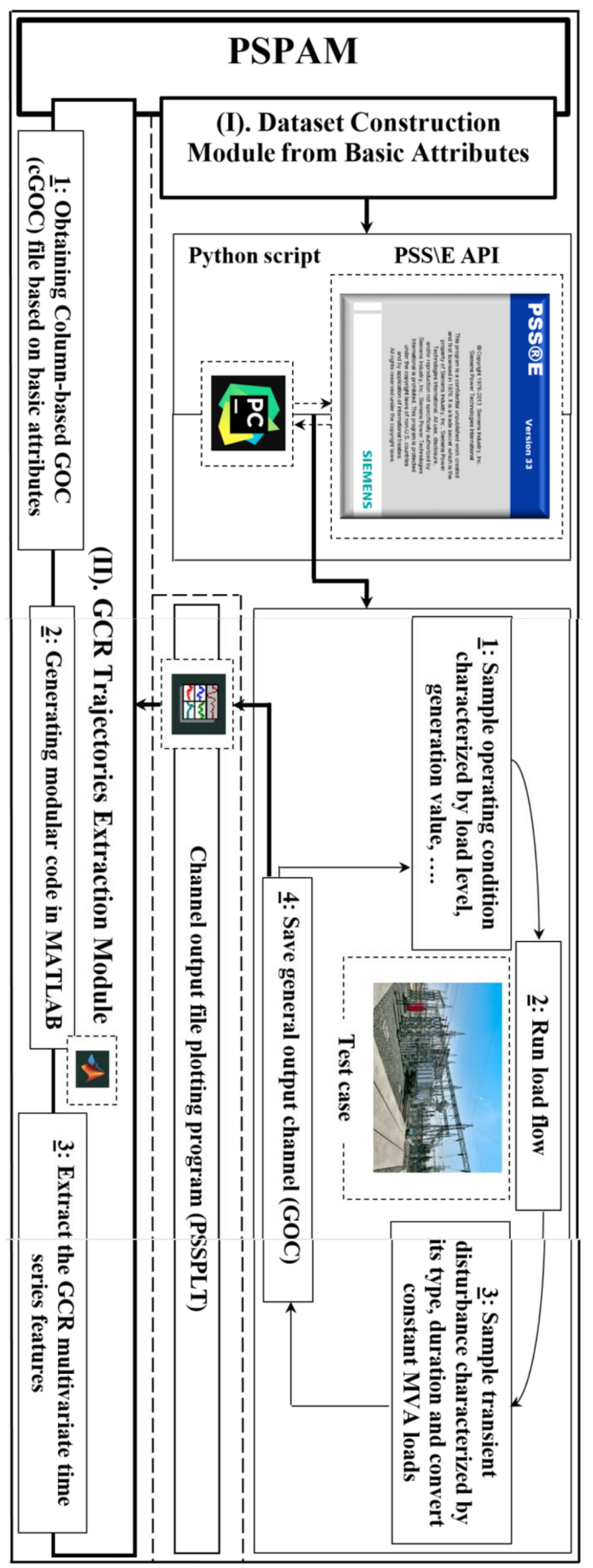

Figure. 2 The PSPAM programming platform for generating GCR multivariate trajectories 
In fact, SVM employs a separating hyperplane with low structural risk in the classification of data and is not linearly separable in feature space. The optimization problem of SVM is defined according to Eq. (3):

$$
\begin{gathered}
\alpha^{*}=\arg \min _{\alpha} \frac{1}{2} \sum_{i=1}^{l} \sum_{j=1}^{l} \alpha_{i} \alpha_{j} y_{i} y_{j} K\left(x_{i} \cdot x_{j}\right)- \\
\sum_{k=1}^{l} \alpha_{k}
\end{gathered}
$$

Where $K\left(x_{i} \cdot x_{j}\right)$ is kernel function performing non-linear mapping into feature space; the constraints are:

$$
0 \leq \alpha_{i} \leq C . \quad \sum_{j=1}^{l} \alpha_{i} y_{i}=0 . \quad i . j=1 . \ldots . l
$$

We can determine the optimal separating hyperplane in feature space solving Eq. (3) with constraints of Eq. (4):

$$
\begin{gathered}
f(x)=\operatorname{sgn}\left(\sum_{i \in S} \alpha_{i} y_{i} K\left(x_{i} \cdot x\right)+b\right) ; \\
b=\frac{1}{S} \sum_{i \in S}\left[y_{i}-\sum_{j} \alpha_{j} y_{j} K\left(x_{j} \cdot x_{i}\right)\right]
\end{gathered}
$$

\subsubsection{Kernel function selection in SVM}

One of the main factors in the SVM algorithm that is affected its performance is the kernel function selection problem. In fact, since we used SVM in the classification process for TSP, the choice of kernel function against $k$-variate feature space is a very important factor. In fact, selecting a proper distance metric for computing distances between trajectory-type objects positively influenced the classification performance. Historical distance metric like Euclidean and Manhattan which aligns the $i$ th point on one trajectory with the $i$ th point on another will produce a poor similarity in score distance and would affect the learning model in terms of TSA. The dynamic time warping (DTW) [26] is a method for finding an optimal match between two given sequences. The DTW has advantages over the Euclidean distance in the aspect of its elastic and robust matching; therefore, it is used in forming the distance matrix of SVM. In this paper, it is tempting to substitute DTW distance for Euclidean distance in the Gaussian RBF kernel and plug it into SVM for sequence classification. The DTW in Gaussian RBF (GRBF) kernel called the DTW-GRBF kernel as $K\left(x_{i} \cdot x_{j}\right)$ in Eq. (3) is defined as Eq. (6):

$$
K\left(x \cdot x^{\prime}\right)=\exp \left(-\frac{\left[\operatorname{distance}^{D T W}\left(A_{1}^{p} \cdot B_{1}^{q}\right)\right]^{2}}{2 \sigma^{2}}\right)
$$

$$
\begin{gathered}
\operatorname{distance}^{D T W}\left(A_{1}^{p} \cdot B_{1}^{q}\right)=d(a(p) \cdot b(q))+ \\
\operatorname{Min}\left(\begin{array}{c}
\text { distance }{ }^{D T W}\left(A_{1}^{p-1} \cdot B_{1}^{q}\right) \\
\text { distance }^{D T W}\left(A_{1}^{p-1} \cdot B_{1}^{q-1}\right) \\
\text { distance }^{D T W}\left(A_{1}^{p} \cdot B_{1}^{q-1}\right)
\end{array}\right)
\end{gathered}
$$

Where $d(.,$.$) is L^{p}$ norm and $A_{1}^{p}$ is a sequence with discrete index varying between 1 and $p$.

\section{Experimental design}

\subsection{Preliminaries of the transient simulation}

In our transient studies, two test cases are considered to evaluate the performance of the proposed framework on TSP. The single-line diagram of SAVNW and IEEE-24 test systems are shown in Fig. 4(a) and Fig 4(b), respectively. For more details on the structure diagram of SAVNW and IEEE-24, refer to [27] and [28], respectively. Also, we consider three types of setting to generate severe transient data. In fact, the triple options make it possible for the real evaluation of predictive model performance on TSP. The concise description of triple options is shown in Table 4. For example, fluctuations of total QELEC to the total of max QELEC ( $\mathrm{F}_{8}$ in Table 1) of SAVNW test case based on quantifying triple-options are shown in Fig. 5 (e.g., yellow: stable univariate and blue: unstable univariate).

Table 4. Descriptions of triple-option to simulate severe transient

\begin{tabular}{ll}
\hline \multicolumn{1}{c}{ Option } & \multicolumn{1}{c}{ Descriptions } \\
\hline fault type & including: $N-1$ and $N-k$ criteria; failure of any one or \\
& $k$ components. \\
convert load: convert the constant MVA & is an array of four elements: [1] IP; constant current \\
load to a specified mixture of the constant & active load, [2] YP; constant admittance active load, \\
current and constant admittance load & [3] IQ; constant current reactive load, and [4] YQ; \\
characteristics. & constant admittance reactive load. \\
simulation time & fault duration time, fault clearing time, and \\
& simulation last for $s$ seconds after the fault clearance. \\
\hline
\end{tabular}




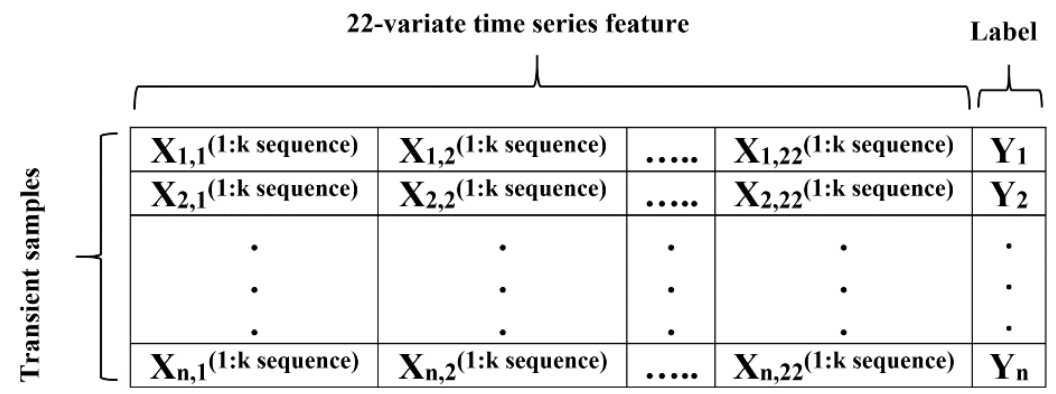

Figure. 3 Transient dataset for TSA

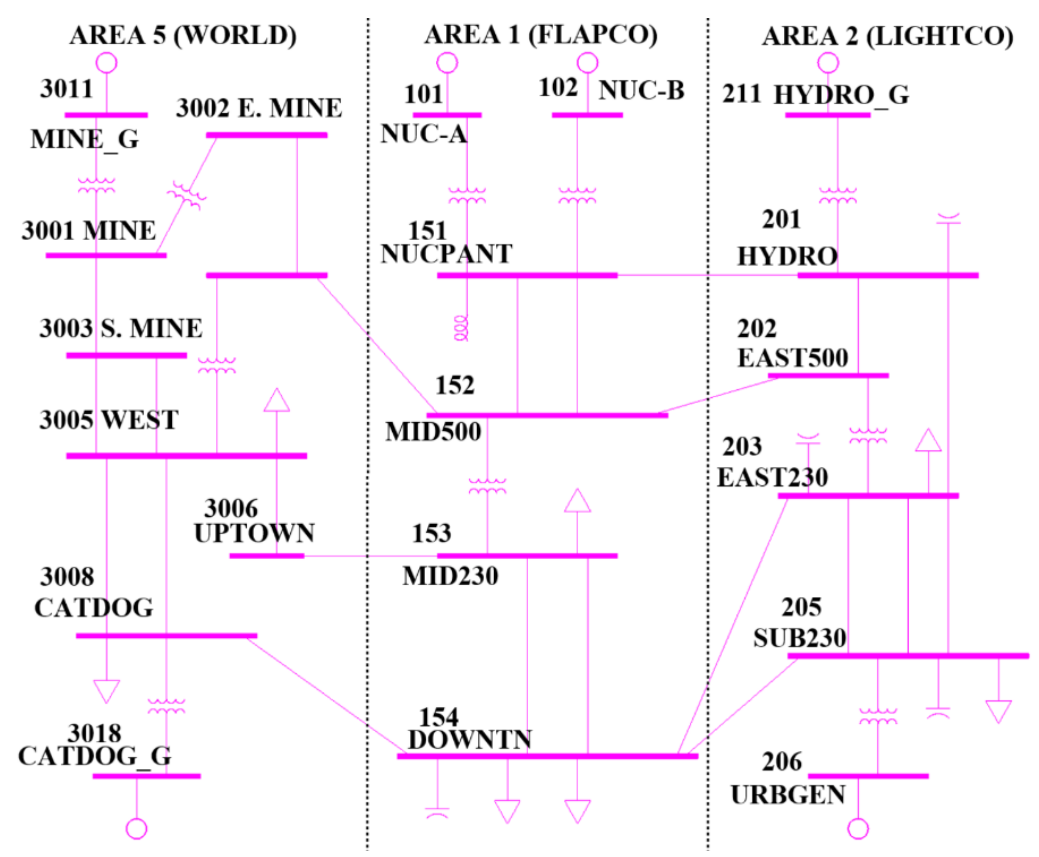

(a)

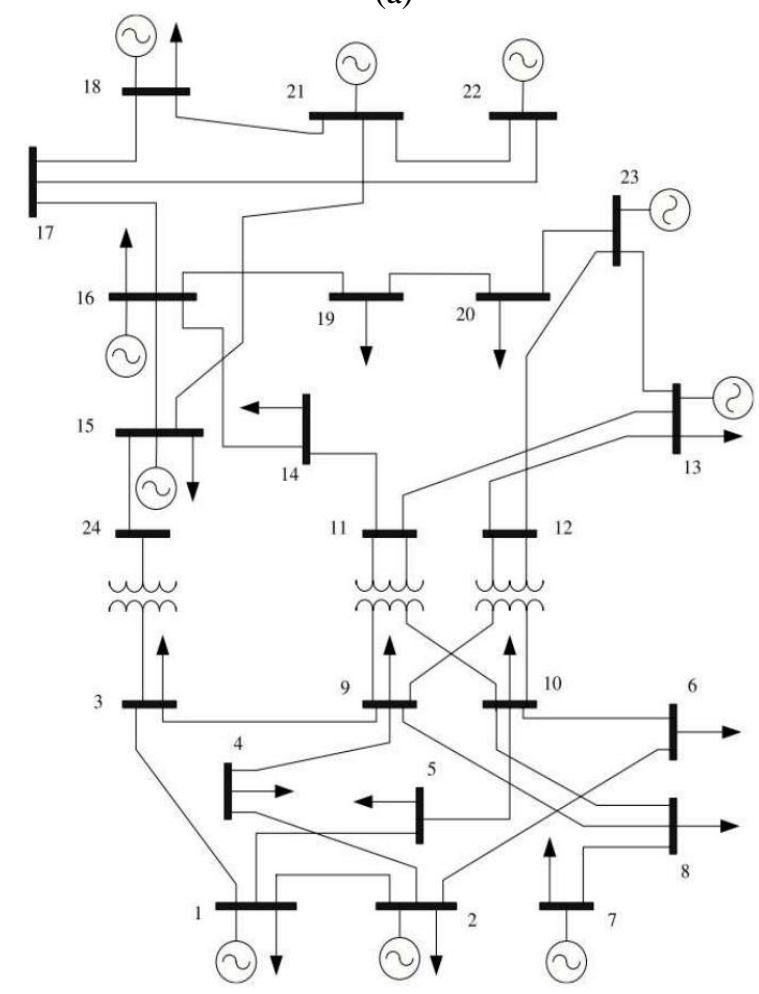

(b)

Figure. 4: (a) One-line diagram of SAVNW and (b) One-line diagram of IEEE-24 [28] 


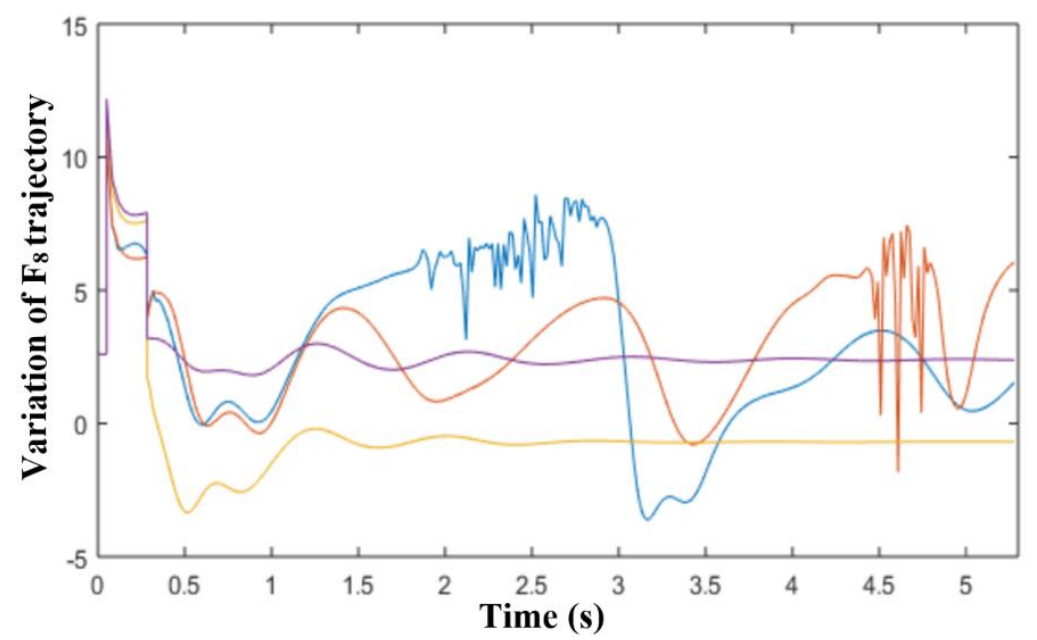

Figure. 5 The stable and unstable fluctuations of F8 on SAVNW test system

\subsection{Performance evaluation of the proposed framework}

In this section, we evaluate the performance of the DTW-GRBF SVM classifier for TSP based on extracted GCR multivariate trajectories. Also, to compare the performance of DTW-GRBF SVM with the other kernel-based SVM for TSP, the GRBF kernel plugged into SVM which is used in [16-20] is applied on GCR trajectories (Eq. (7)):

$$
K\left(x \cdot x^{\prime}\right)=\exp \left(-\frac{|| x-x^{\prime}||^{2}}{2 \sigma^{2}}\right)
$$

Where $\left\|x-x^{\prime}\right\|^{2}$ is squared Euclidean distance between the two trajectory features.

Furthermore, the following metrics (See Table 5) are considered for measuring the performance of the predictive model. Also, to test the performance of elastic and non-elastic SVM classifier on TSP, 5fold cross validation which involves 5 iterations was considered. Average the 5 classifiers performance (Acc, TPR, and TNR) is obtained from 5 splits.

Also, to select optimal pairs of parameters for classifiers $(C$ and $\sigma$ ), the parameters $C$ and $\sigma$ are selected from $\left\{2^{\mathrm{i}} \mid \mathrm{i}=0,1,2, \ldots, 7\right\}$ and $\left\{2^{\mathrm{j}} \mid \mathrm{j}=-4,-3\right.$, $\ldots, 3\}$, respectively. In this way, the maximum value of accuracy metrics for each fold is obtained.

Table 5. The performance metrics

\begin{tabular}{ll}
\hline Metrics & Descriptions \\
\hline Accuracy & Acc $=(\mathrm{TP}+\mathrm{TN}) /(\mathrm{TP}+\mathrm{TN}+\mathrm{FP}+\mathrm{FN})$ \\
Sensitivity & TPR=TP/(TP+FN) \\
Specificity & $\mathrm{TNR}=\mathrm{TN} /(\mathrm{TN}+\mathrm{FP})$ \\
\hline
\end{tabular}

Symbols; P: stable sample, N: unstable sample,

$\mathrm{T}$ : predicted correctly, F: predicted incorrectly

\subsubsection{Timesaving on TSP}

Timesaving on TSP to perform appropriate corrective action [2] is a basic principle on power system transient control strategy. In fact, a fast and accurate approach is a decisive requirement for TSA tasks. Hence, using the partial of transient fluctuations (PoT) after clearing the fault for evaluating the learning process was considered in this section. As can be seen in Table 6 and 7, training and testing procedures with the length of PoT in 15, 25, and 35 cycles after clearing the fault were conducted using GRBF SVM and DTW-GRBF SVM on test systems. The increasing size of PoT from 15 to 35 affects (positive manner) the classification performance of the predictive model in most cases. Furthermore, comparing the performance of two classifiers on the same test system regarding different PoT showed the fact that non-linear alignment in DTW outperforms point to point alignment in Euclidean distance on test systems in most cases. According to Table 6 and 7, DTW-GRBF SVM had high-performance based on triple metrics (row labelled as mean), while GRBF SVM had the low-performance for TSP. However, regarding obtained results of the mean (measure), GRBF SVM has high TNR (only PoT: 15 cycles) on IEEE-24 and high TNR (only PoT:15 cycles) on SAVNW than DTW-GRBF SVM. Due to DTW depended on increasing the length of PoT for nonlinear alignment in feature space, reducing PoT from35 to 15 sometimes affects the performance of DTW-GRBF SVM in a negative manner. Overall, the choice of DTW distance plugged into kernel machine that induces DTW-GRBF SVM outperforms GRBF SVM for TSP. Table 8 offers the concise report of Tables $6 \& 7$ for readers at a glance regarding the performance of GRBF SVM 
Table 6. Result of TSP based on GRBF SVM with different PoT

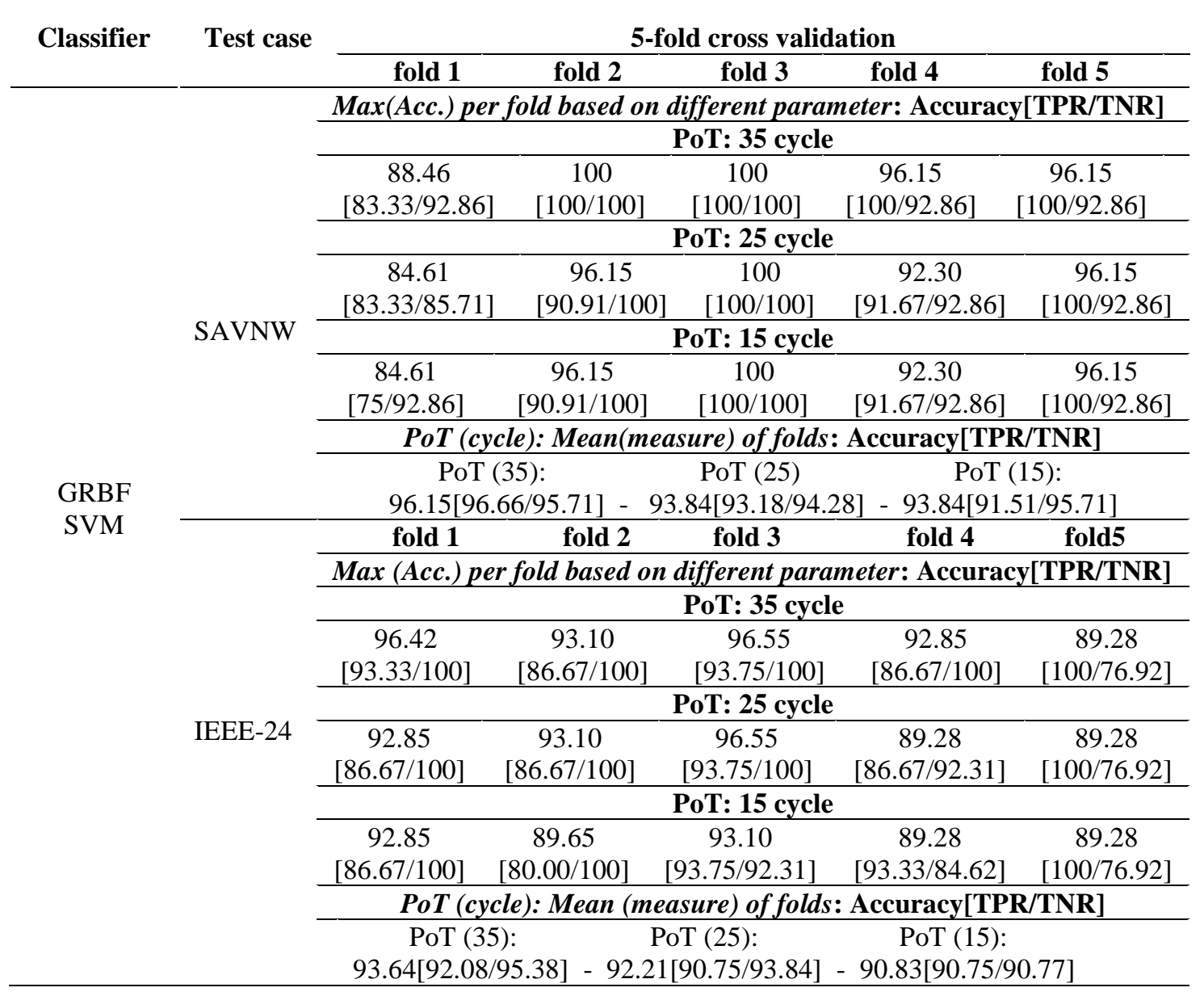

and DTW-GRBF SVM on TSP. As can be seen in Table 8, DTW-GRBF SVM is the winner algorithm in most of the existing scenarios in the presence of the triple metrics and the test grids. Fig. 6 shows the performance variation (Acc) of GRBF SVM with triple $\operatorname{PoT}(15,25$, and 35 cycles) and different learning parameters grouped by folds on the SAVNW test system. As can be seen in Fig. 6, the Acc surface of the 35 cycles PoT is dominant against other surface (15 and 25 cycle) in most points on folds. Also, Fig. 7 shows the performance variation (Acc) of DTW-GRBF SVM with length of PoT in 15 cycles regarding different learning parameters per fold on the IEEE-24 test system.

\subsubsection{Impact of PoT on the TSP processing time}

The processing time for TSP based on different PoT is shown in Table 9. The processing time for TSP is comprised of the different PoT after clearing the fault and the prediction time of the classifier. The result shows the GRBF SVM has a lower processing time for TSP. However, if PSO requests to achieve better performance (triple metric) than GRBF SVM for TSP, should be switched to DTW-
GRBF SVM regarding PoT 35 \& 25 cycles on SAVNW and IEEE-24 that gained 927.6 milliseconds (< $1 \mathrm{~s})$. Also, using DTW-GRBF SVM regarding PoT 25 cycles on SAVNW and IEEE-24 is an acceptable processing time (664.4 milliseconds) along with high performance in all metrics. Furthermore, DTW-GRBF SVM regarding 15 cycles PoT on IEEE-24 and SAVNW (ignoring only $1.43 \%$ less TNR than GRBF SVM in the same PoT) has affordable processing time (429.6 milliseconds) along with high performance.

\subsubsection{Comparison of the proposed strategy (DTW- GRBF SVM+GCR features) with other frameworks}

In addition to comparing the proposed DTWGRBF SVM with the GRBF-SVM used in [16-20] for TSP based on defined multivariate trajectory features in sub-section 3.2.1-3.2.2, for more analysis on the effectiveness of our framework (DTW-GRBF SVM accompanied with GCR 22-variate time series), we compared the proposed framework with other strategies (learning method + defined features) in $[14,15]$. For more technical details on the offered packages (proposed learning algorithm + 
Table 7. Result of TSP based on DTW-GRBF SVM with different PoT

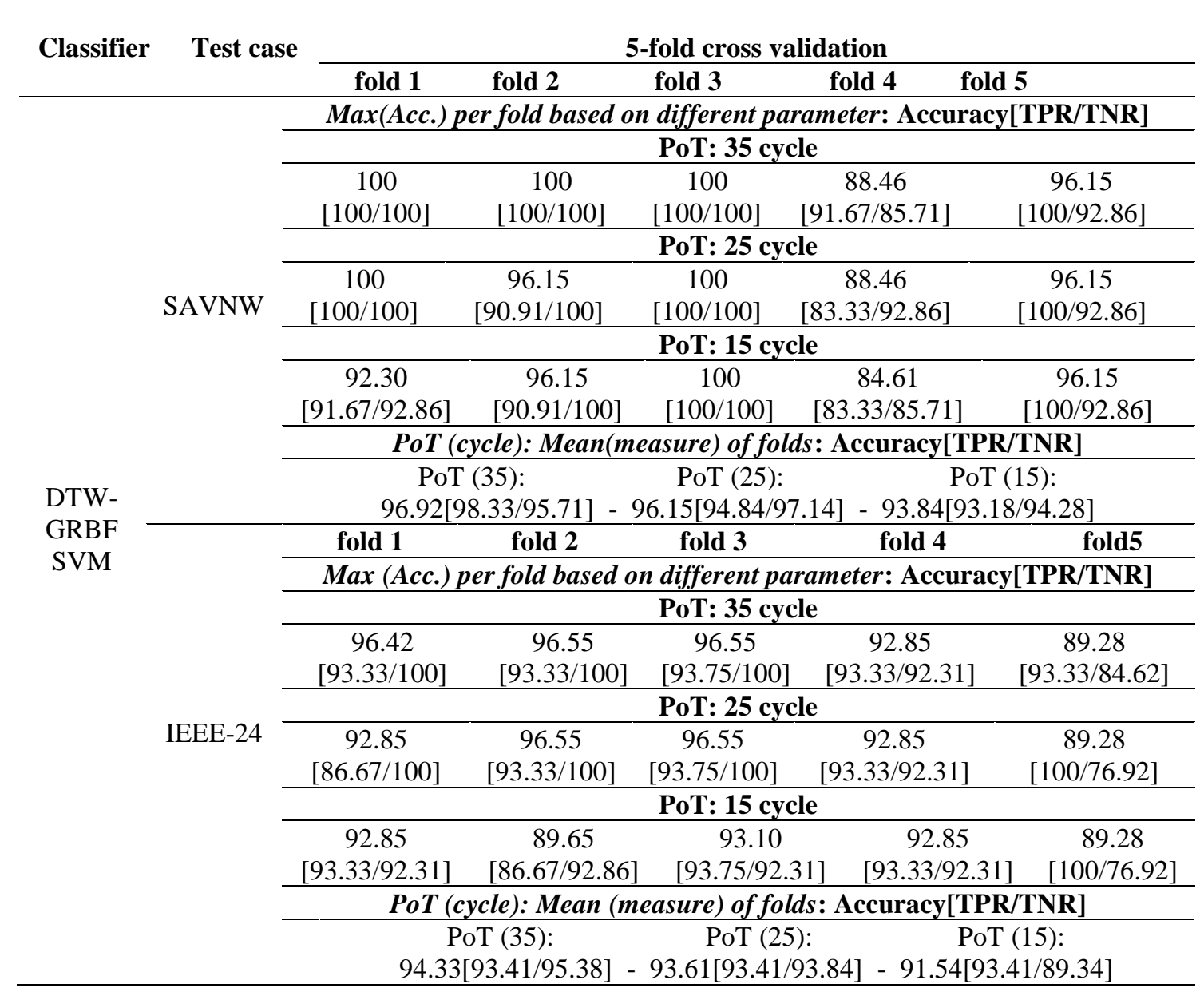

Table 8. The concise report of methods performance based on results of Table $6 \& 7$

\begin{tabular}{|c|c|c|c|c|c|}
\hline Test case & PoT & Method & Acc & TPR & TNR \\
\hline \multirow{6}{*}{ IEEE-24 } & \multirow[t]{2}{*}{35 cycles } & GRBF SVM & $\mathrm{L}$ & $\mathrm{L}$ & $\mathrm{D}$ \\
\hline & & DTW-GRBF SVM & $\mathrm{W}$ & $\mathrm{W}$ & $\mathrm{D}$ \\
\hline & \multirow[t]{2}{*}{25 cycles } & GRBF SVM & $\mathrm{L}$ & $\mathrm{L}$ & $\mathrm{D}$ \\
\hline & & DTW-GRBF SVM & $\mathrm{W}$ & $\mathrm{W}$ & $\mathrm{D}$ \\
\hline & \multirow[t]{2}{*}{15 cycles } & GRBF SVM & $\mathrm{L}$ & $\mathrm{L}$ & $\mathrm{W}$ \\
\hline & & DTW-GRBF SVM & $\mathrm{W}$ & $\mathrm{W}$ & $\mathrm{L}$ \\
\hline \multirow{6}{*}{ SAVNW } & \multirow[t]{2}{*}{35 cycles } & GRBF SVM & $\mathrm{L}$ & $\mathrm{L}$ & $\mathrm{D}$ \\
\hline & & DTW-GRBF SVM & $\mathrm{W}$ & $\mathrm{W}$ & $\mathrm{D}$ \\
\hline & \multirow[t]{2}{*}{25 cycles } & GRBF SVM & $\mathrm{L}$ & $\mathrm{L}$ & $\mathrm{L}$ \\
\hline & & DTW-GRBF SVM & $\mathrm{W}$ & $\mathrm{W}$ & $\mathrm{W}$ \\
\hline & \multirow[t]{2}{*}{15 cycles } & GRBF SVM & $\mathrm{D}$ & $\mathrm{L}$ & $\mathrm{W}$ \\
\hline & & DTW-GRBF SVM & $\mathrm{D}$ & $\mathrm{W}$ & $\mathrm{L}$ \\
\hline Total Results (\# wins) & Acc (6 match) & TPR (6 match) & \multicolumn{3}{|c|}{ TNR (6 match) } \\
\hline GRBF SVM & 0 & 0 & \multicolumn{3}{|c|}{2} \\
\hline DTW-GRBF SVM & 5 & 6 & \multicolumn{3}{|c|}{1} \\
\hline \multicolumn{6}{|c|}{ DTW-GRBF SVM (12) : (2) GRBF SVM } \\
\hline
\end{tabular}

W: won, D: draw, L: Loss 


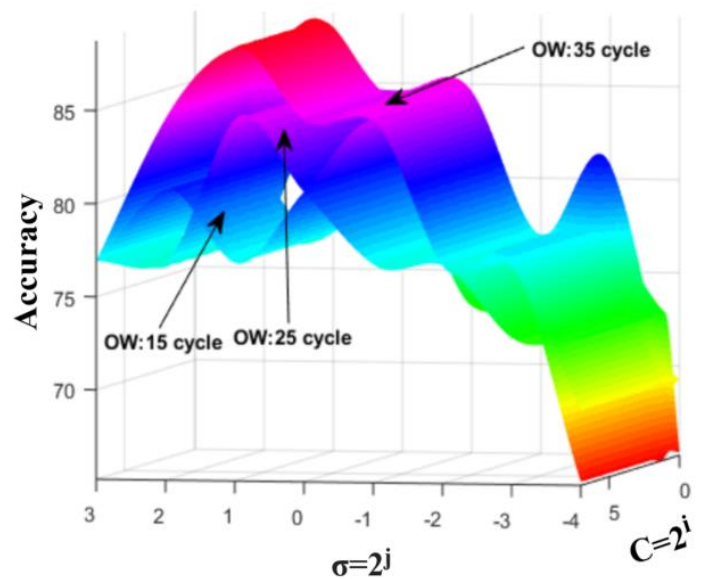

(a)

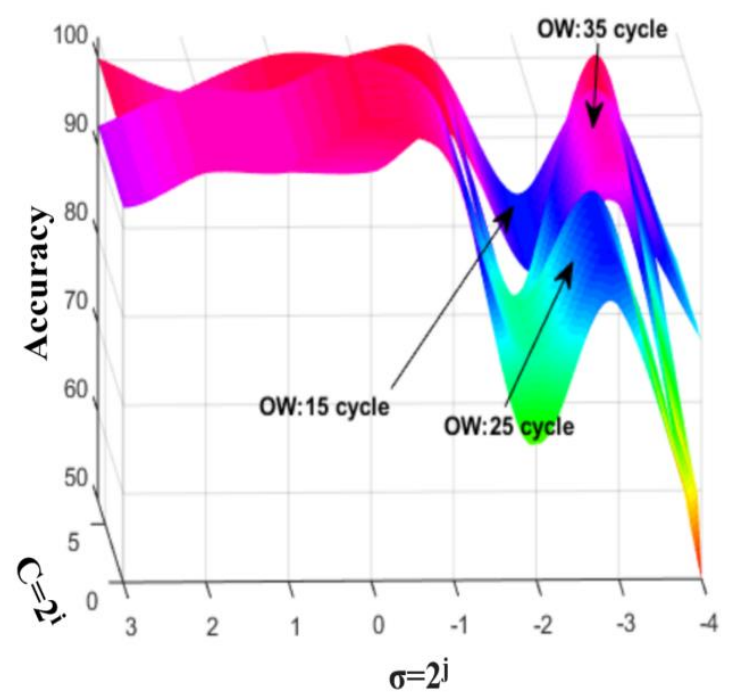

(c)

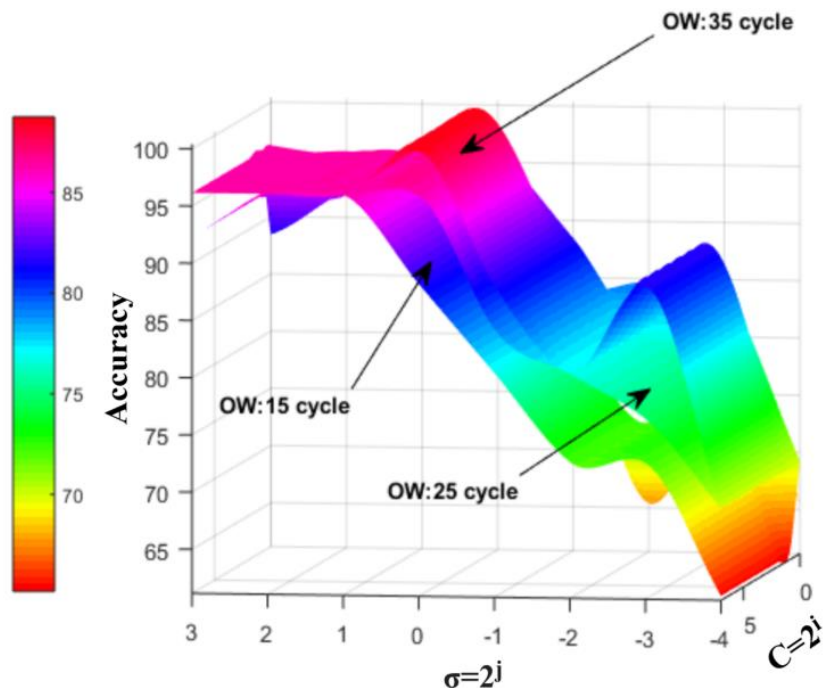

(b)
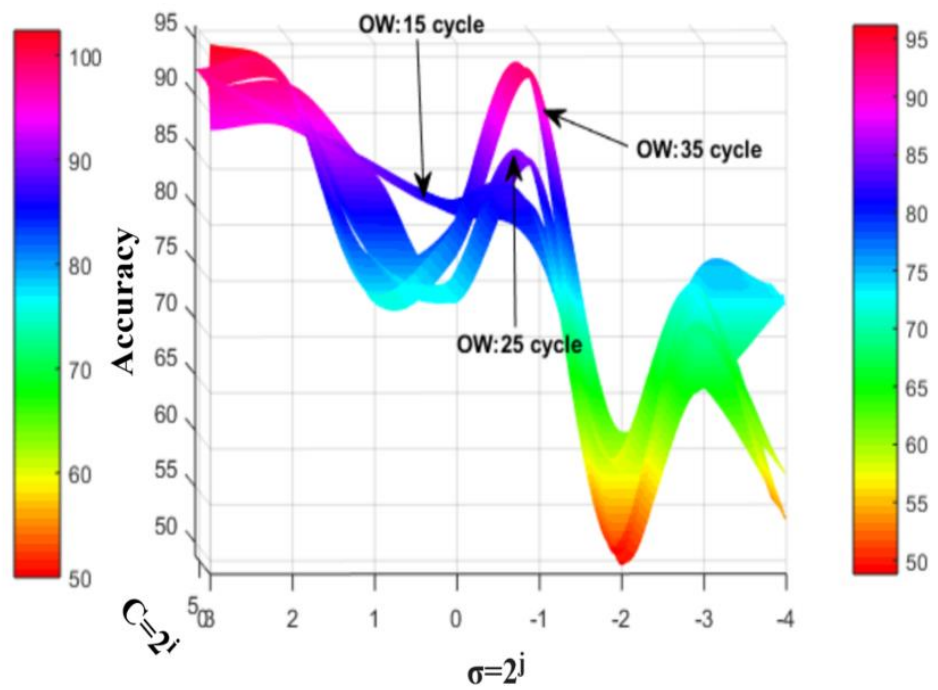

(d)

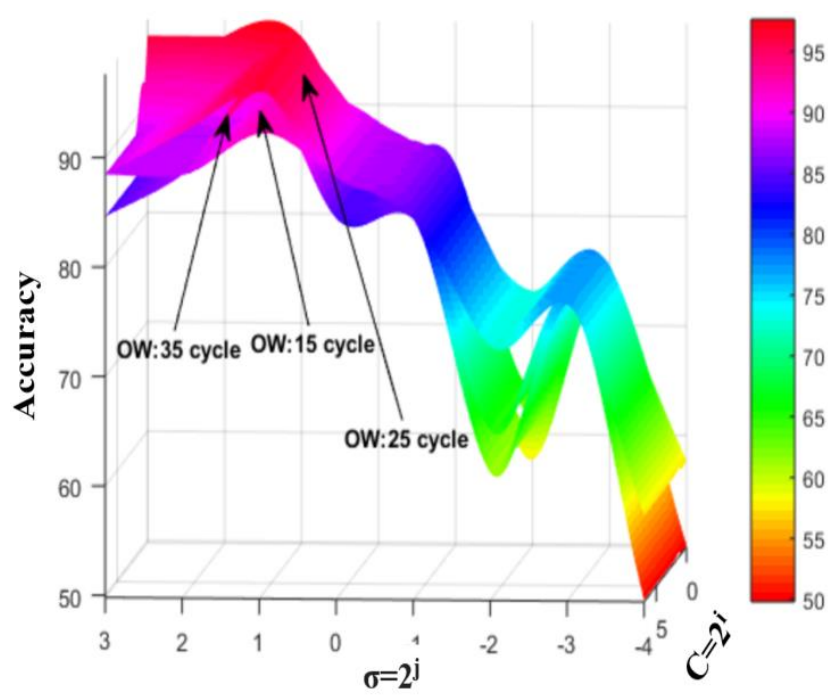

(e)

Figure. 6 Illustration of GRBF SVM performance with different PoT and learning parameters; grouped by folds on SAVNW: (a) Fold 1, (b) Fold 2, (c) Fold 3, (d) Fold 4, and (e) Fold 5 


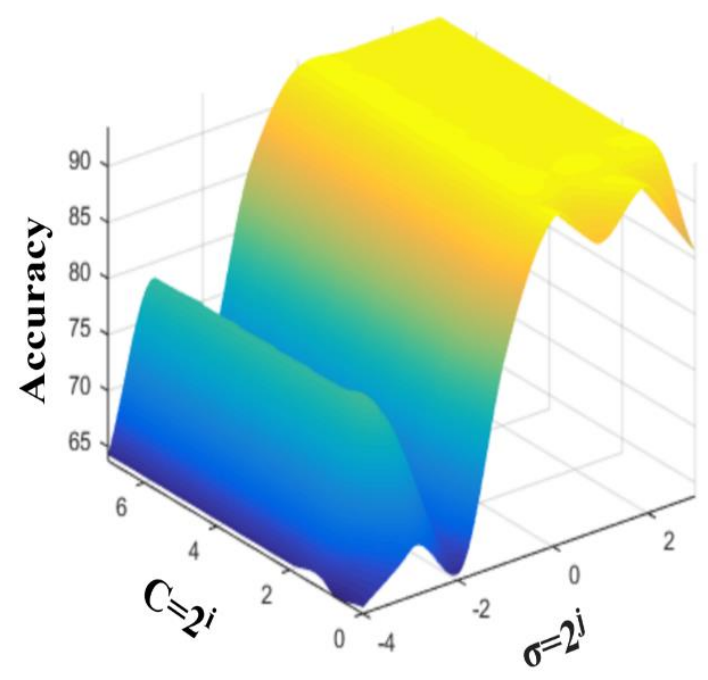

(a)

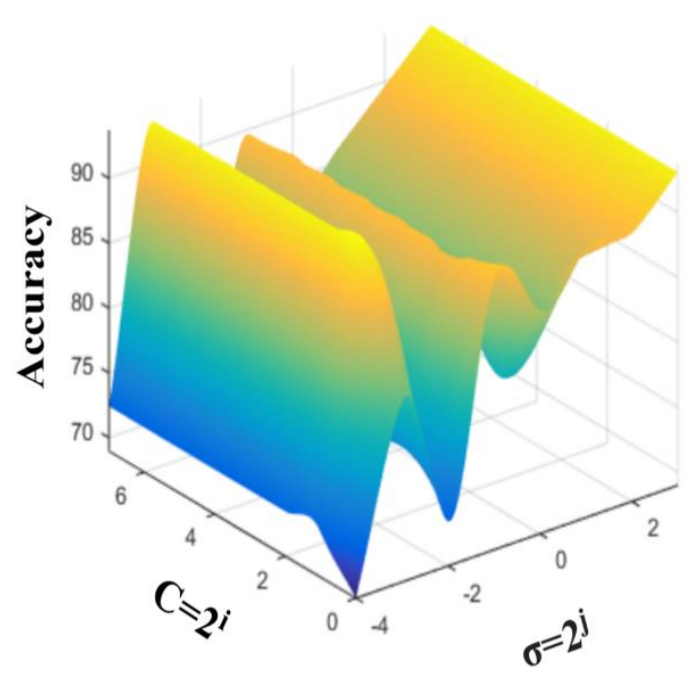

(c)
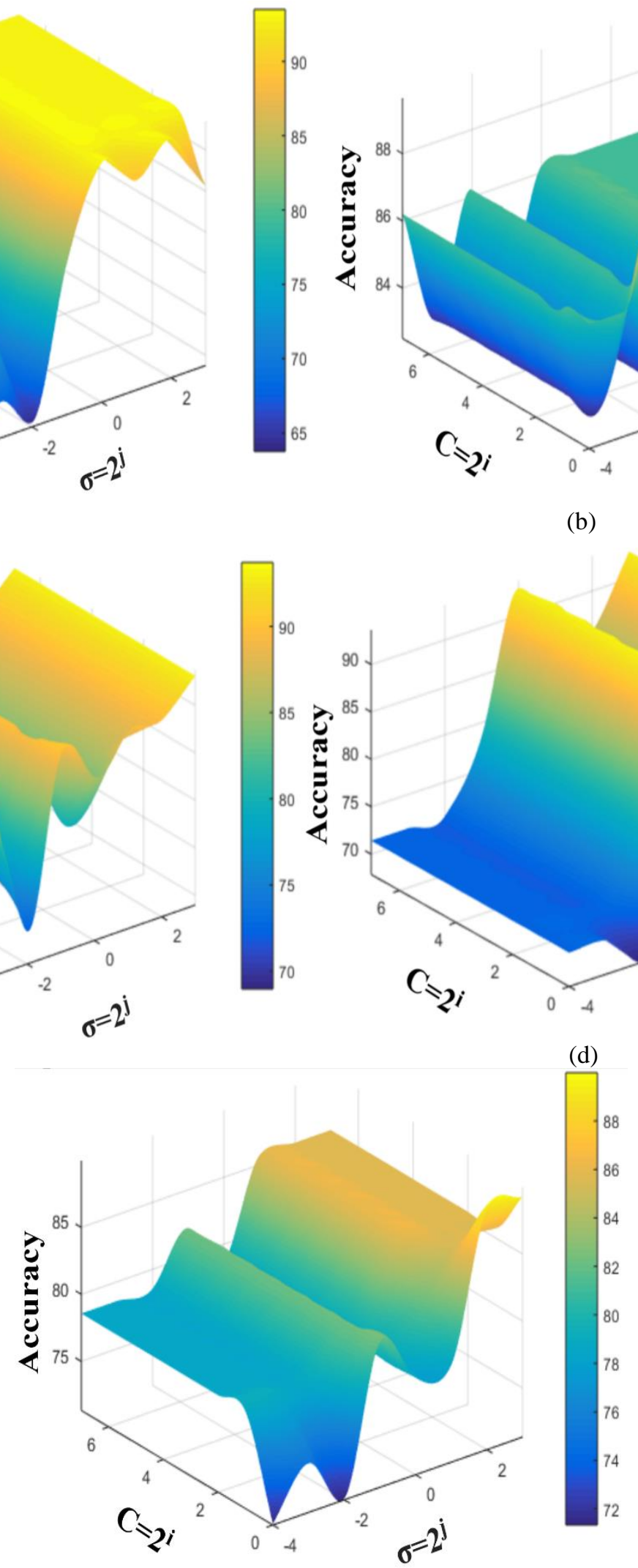

(e)

(d)

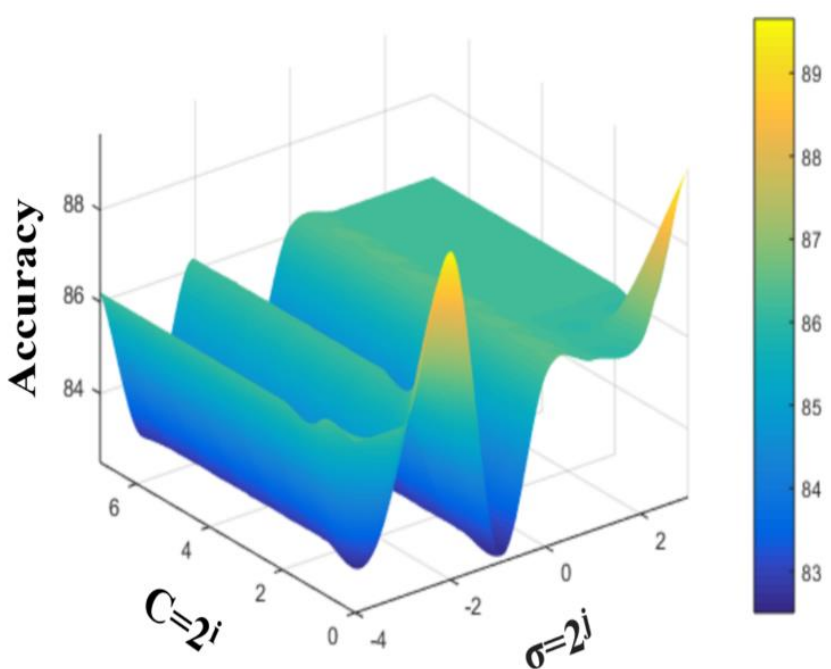

(b)

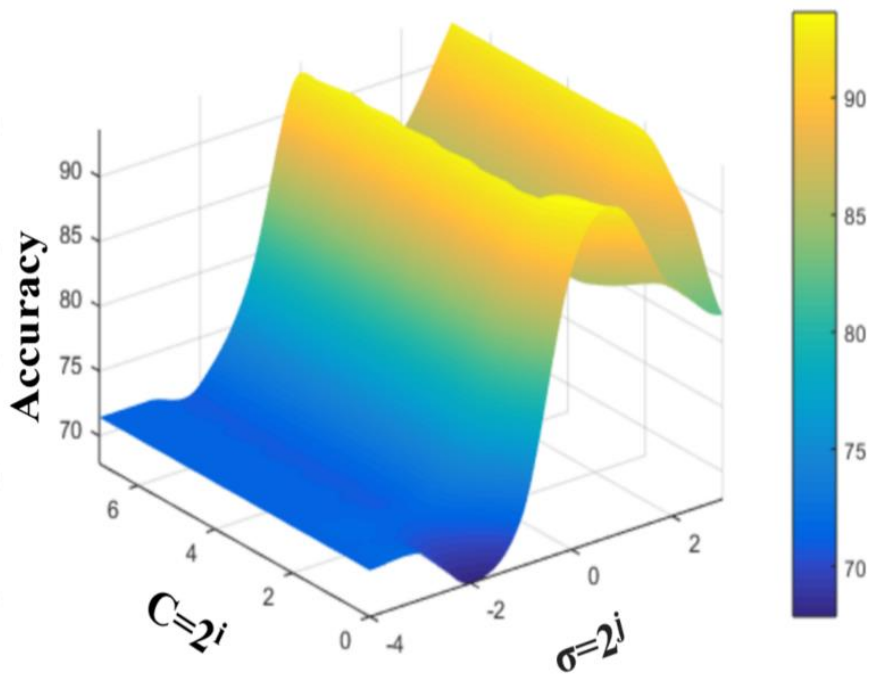

Figure. 7 Illustration of DTW-GRBF SVM performance with different parameters on IEEE-24 bus (PoT: 15 cycle): (a) Fold 1, (b) Fold 2, (c) Fold 3, (d) Fold 4, and (e) Fold 5 


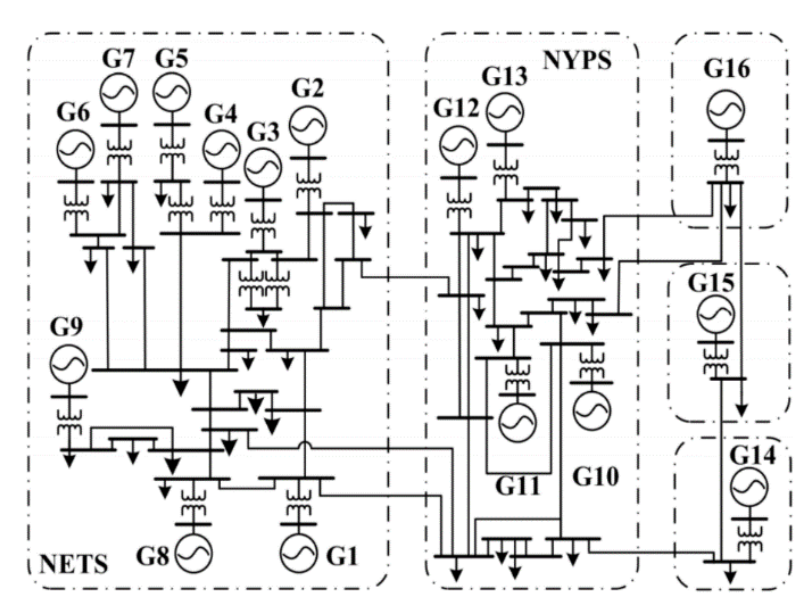

Figure. 8 One-line diagram of NETS-NYPS [30]

Table 9. Processing time with different PoT

\begin{tabular}{|c|c|c|c|}
\hline Classifier & $\begin{array}{l}\text { PoT in } \\
\text { cycle }\end{array}$ & $\begin{array}{l}\text { PoT in } \\
\text { second }\end{array}$ & Processing Time $^{\mathrm{a}}$ \\
\hline \multirow{3}{*}{$\begin{array}{c}\text { GRBF } \\
\text { SVM }\end{array}$} & 15 & 0.2505 & $\begin{array}{l}250.5 \mathrm{~ms}^{\mathrm{b}}+16.06 \mathrm{~ms}= \\
266.56 \mathrm{~ms}\end{array}$ \\
\hline & 25 & 0.4175 & $\begin{array}{l}417.5 \mathrm{~ms}+16.23 \mathrm{~ms}= \\
433.73 \mathrm{~ms}\end{array}$ \\
\hline & 35 & 0.5845 & $\begin{array}{l}584.5 \mathrm{~ms}+17.56 \mathrm{~ms}= \\
602.06 \mathrm{~ms}\end{array}$ \\
\hline \multirow{3}{*}{$\begin{array}{l}\text { DTW- } \\
\text { GRBF } \\
\text { SVM }\end{array}$} & 15 & 0.2505 & $\begin{array}{l}250.5 \mathrm{~ms}+179.1 \mathrm{~ms}= \\
429.6 \mathrm{~ms}\end{array}$ \\
\hline & 25 & 0.4175 & $\begin{array}{l}417.5 \mathrm{~ms}+246.9 \mathrm{~ms}= \\
664.4 \mathrm{~ms}\end{array}$ \\
\hline & 35 & 0.5845 & $\begin{array}{l}584.5 \mathrm{~ms}+343.1 \mathrm{~ms}= \\
927.6 \mathrm{~ms}\end{array}$ \\
\hline
\end{tabular}

a Processing Time $=$ PoT + prediction time $;{ }^{\mathrm{b}}$ millisecond

Table 10. Comparing the performance of triple packages for TSA

\begin{tabular}{|cccc|}
\hline $\begin{array}{c}\text { Package } \\
\text { (learning algorithm } \\
\text { + defined features) }\end{array}$ & Acc & TPR & TNR \\
\hline $\begin{array}{c}\text { (I) DTW-GRBF SVM } \\
\text { + GCR 22-variate } \\
\text { trajectory features } \\
\text { (our package) }\end{array}$ & 98.66 & 97 & 100 \\
\hline $\begin{array}{c}\text { (II) GRBF-SVM + } \\
\text { reactive power-based } \\
\text { two variate time } \\
\text { series (RP2vTS) [14] }\end{array}$ & 95.84 & 94.44 & 97.30 \\
\hline $\begin{array}{c}\text { (III) twin convolutional } \\
\text { SVM + 8-variate }\end{array}$ & 85.71 & 100 & 71.43 \\
trajectory features \\
[15]
\end{tabular}

defined transient features), refer to [14] and [15]. Also, we addressing the performance evaluation of triple packages (our framework and $[14,15]$ ) against the New England-New York interconnection system (NETS-NYPS) [29], which has the size of topology larger than SAVNW and IEEE-24 (Fig. 8). As can be seen in Table 10, our proposed framework including DTW-GRBF SVM and 22-variate geometric time-series features outperforms the presented framework in [14] based on triple metrics on the NETS-NYPS grid case. Also, our proposed package has high performance on Acc and TNR than the proposed package in [15]. Our strategy only $3 \%$ less TPR than the proposed package in [15]. Also, our strategy has a lower processing time than the presented frameworks in $[14,15]$ for TSA.

\section{Conclusion}

In this paper, our proposed framework is offered for transient stability status prediction as a companion during the online procedure of real-time TSA. According to the proposed programming platform, first, basic reasons involved in the definition of GCR 22-variate time series features are discussed. After defining GCR features, transient data generation is comprised of two phases: the obtained transient data based on GOC file and GCR trajectories extraction. To this end, we triangulated Python script, SIEMENS PSS/E API, and Matlab for generating required data for transient analysis. Next, the elastic SVM classifier as a learning method is conducted for transient stability status prediction. Finally, the model trained using GCR trajectories is tested for evaluating the performance of the proposed framework. The classification accuracy based on DTW-GRBF SVM near $97 \%$ for SAVNW and $95 \%$ for IEEE-24 test systems. According to obtained results, DTW-GRBF SVM has a high performance based on triple metrics in 35 \& 25 cycles PoT on test cases, while GRBF SVM has high TNR (only 15 cycles PoT) on IEEE-24 and TNR (only 15 cycles PoT) on SAVNW. Also, GRBF SVM has a short processing time (266.56 milliseconds in 15 cycles) for TSP, while DTWGRBF SVM regarding 35 cycles PoT has an acceptable processing time (927.6 milliseconds) along with high performance (all metrics) in both test case. Also, for more analysis on the effectiveness of the proposed framework (DTWGRBF SVM + GCR features), we compare the proposed strategy with other frameworks on the NETS-NYPS grid case. The obtained results show that the proposed method accompanied with 22variate GCR features outperforms other frameworks (method+features) for TSP. Overall, the results show that our proposed methodology will be applicable as a synergetic suite on severe transient scenarios when confronted by non-identically obtained GCR trajectories in offline and online parts 
for real-time TSA. For future work, the environmental factors as an integral part of transient studies should be considered for TSA. In fact, communication channel failure and noisy data are the most significant issue negatively influenced the quality of transient data and its caused to lowperformance TSP. Hence, applying a hyperplanebased approach with a proper kernel specified for incomplete trajectories should be considered as a vital task in future transient studies.

\section{Conflicts of Interest}

The authors declare no conflict of interest.

\section{Author Contributions}

The paper conceptualization, methodology, software, validation, formal analysis, investigation, resources, data curation, supervision, and project administration done by 1 st author. Also, writingoriginal draft preparation, writing-review and editing, visualization done by 2 nd author.

\section{References}

[1] P. Kundur, J. Paserba, V. Ajjarapu, G. Andersson, A. Bose, C. Canizares, N. Hatziargyriou, D. Hill, A. Stankovic, C. Taylor, T. V. Cutsem, and V. Vittal, "Definition and Classification of Power System Stability IEEE/CIGRE Joint Task Force on Stability Terms and Definitions", IEEE Transactions on Power Systems, Vol. 19, No. 3, pp. 1387-1401, 2004.

[2] M. Pavella, D. Ernst, and D. R. Vega, Transient Stability of Power Systems: A Unified Approach to Assessment and Control, Springer, Boston, M.A. 2000.

[3] Y. Zhou, J. Wu, L. Hao, L. Ji, and Z. Yu, "Transient Stability Prediction of Power Systems Using Post-Disturbance Rotor Angle Trajectory Cluster Features", Electric Power Component and Systems, Vol. 44, No. 17, pp. 1879-1891, 2016.

[4] L. Ji, J. Wu, Y. Zhou, and L. Hao, "Using Trajectory Clusters to Define the Most Relevant Features for Transient Stability Prediction Based on Machine Learning Method", Energies, Vol. 9, No. 11, p. 898, 2016.

[5] X. Li, Z. Zheng, L. Wu, R. Li, J. Huang, X. Hu, and P. Guo, "A Stratified Method for LargeScale Power System Transient Stability Assessment Based on Maximum Relevance
Minimum Redundancy Arithmetic", IEEE Access, Vol. 7, pp. 61414-61432, 2019.

[6] A. F. D. Alzate, J. E. C. Becerra, and J. F. V. Sierra, "Transient Stability Prediction for RealTime Operation by Monitoring the Relative Angle with Predefined Thresholds", Energies, Vol. 12, No. 5, p. 838, 2019.

[7] J. C. G. Torres, G. Damm, V. Costan, A. Benchaib, and F. L. Lagarrigue, "A Novel Distributed Supplementary Control of MultiTerminal VSC-HVDC Grids for Rotor Angle Stability Enhancement of AC/DC Systems", IEEE Transactions on Power Systems, Vol. 36, No. 1, pp. 623-634, 2021.

[8] S. Dasgupta, M. Paramasivam, U. Vaidya, and V. Ajjarapu, "PMU-Based Model-Free Approach for Real-Time Rotor Angle Monitoring", IEEE Transactions on Power Systems, Vol. 30, No. 5, pp. 2818-2819, 2015.

[9] A. Mitra and D. Chatterjee, "Active Power Control of DFIG-Based Wind Farm for Improvement of Transient Stability of Power Systems", IEEE Transactions on Power Systems, Vol. 31, No. 1, pp. 82-93, 2016.

[10] A. Mujcinagic, M. Kusljugic, and E. Nukic, "Wind Inertial Response Based on the Center of Inertia Frequency of a Control Area", Energies, Vol. 13, No. 23, p. 6177, 2020.

[11] J. Zhao, Y. Zhu, and J. Tang, "Transient Voltage and Transient Frequency Stability Emergency Coordinated Control Strategy for the Multi-infeed HVDC Power Grid", In: Proc. of IEEE Power \& Energy Society General Meeting (PESGM), Canada, 2020.

[12] T. Liu and X. Wang, "Transient Stability of Single-Loop Voltage-Magnitude Controlled Grid-Forming Converters", IEEE Transactions on Power Electronics, Vol. 36, No. 6, pp. 61586162, 2021.

[13] F. Milano and Á. O. Manjavacas, "FrequencyDependent Model for Transient Stability Analysis", IEEE Transactions on Power Systems, Vol. 34, No. 1, pp. 806-809, 2019.

[14] S. A. B. Mosavi, "Extracting Discriminative Features in Reactive Power Variations by 1Persistence Parallel Fragmented Hybrid Feature Selection Scheme for Transient Stability Prediction", International Journal of Intelligent Engineering and Systems, Vol. 14, No. 4, pp. 500-513, 2021.

[15] A. B. Mosavi, A. Amiri, and H. Hosseini, "A Learning Framework for Size and Type Independent Transient Stability Prediction of Power System Using Twin Convolutional 
Support Vector Machine", IEEE Access, Vol. 6, pp. 69937-69947, 2018.

[16] F. R. Gomez, A. D. Rajapakse, U. D. Annakkage, and I. T. Fernando, "Support Vector Machine-Based Algorithm for PostFault Transient Stability Status Prediction Using Synchronized Measurements", IEEE Trans. Power System, Vol. 26, No. 3, pp. 14741483, 2011.

[17] L. S. Moulin, A. P. A da Silva, M. A. E. Sharkawi, and R. J. Marks, "Support Vector Machines for Transient Stability Analysis of Large-Scale Power Systems", IEEE Transactions on Power Systems, Vol. 19, No. 2, pp. 818-825, 2004.

[18] D. You, K. Wang, L. Ye, J. Wu, and R. Huang, "Transient Stability Assessment of Power System Using Support Vector Machine with Generator Combinatorial Trajectories Inputs", International Journal of electrical Power \& Energy Systems, Vol. 44, No. 1, pp. 318-325, 2013.

[19] S. Ye, X. Li, X. Wang, and Q. Qian, "Power System Transient Stability Assessment based on AdaBoost and Support Vector machines", In: Proc. of Asia-pacific Power and Energy Engineering Conference, China, 2012.

[20] X. Wang, S. Wu, Q. Li, and X. Wang, "v-SVM for Transient Stability Assessment in Power Systems", In: Proc. of Autonomous Decentralized Systems, China, 2005.

[21] PSS/E 33.4 application program interface (API), Siemens industry, Schenectady, USA, 2013.

[22] PSS/E 33.4 PSSPLT program manual (API), Siemens industry, Schenectady, USA, 2013.

[23] G. Kou, "Dynamic Modeling and Renewable Integration Studies on the U.S. Power Grids", Ph.D. dissertation, Electrical Engineering. Dept., University of Tennessee, Knoxville, 2016, Available:

https://trace.tennessee.edu/utk_graddiss/3865/

[24] C. Cortes and V. Vapnik, "Support Vector Networks", Springer, Vol. 20, No. 3, pp. 272297, 1995.

[25] V. Cherkassky and F. Mulier, Learning from Data: Concepts Theory and Method, WilleyIEEE Press, New Jersey, 2007.

[26] M. Muller, Information Retrieval for Music and Motion, Springer-Verlag, Berlin, 2007.

[27] PSS/E 33.4 PSSPLT program operation manual, Siemens industry, Schenectady, USA, 2013.

[28] C. Ordoudis, P. Pinson, J. M. M. Gonzalez, and M. Zugno, An Updated Version of the IEEE RTS 24-Bus System for Electricity Market and
Power System Operation Studies, Technical University of Denmark, pp. 1-5, 2016.

[29] G. Rogers, Power System Oscillations, Springer, New York, 2000.

[30] Y. Zhou, J. Wu, Z. Yu, L. Ji, and L. Hao, “A Hierarchical Method for Transient Stability Prediction of Power Systems Using the Confidence of a SVM-Based Ensemble Classifier", Energies, Vol. 9, No, 10, p. 778, 2016. 